\title{
On a Microscopic Representation of Space-Time IV*
}

\author{
Rolf Dahm ${ }^{1}$ \\ ${ }^{1}$ Permanent address: beratung für Informationssysteme und Systemintegration, \\ Gärtnergasse 1, D-55116 Mainz, Germany
}

We summarize some previous work on $\mathrm{SU}(4)$ describing hadron representations and transformations as well as its non-compact 'counterpart' SU*(4) being the complex embedding of $\mathrm{SL}(2, \mathbb{H})$. So after having related the 16 -dim Dirac algebra to $\mathrm{SU} *(4)$, on the one hand we have access to real, complex and quaternionic Lie group chains and their respective algebras, on the other hand it is of course possible to relate physical descriptions to the respective representations. With emphasis on the common maximal compact subgroup USp(4), we are led to projective geometry of real 3-space and various transfer principles which we use to extend previous work on the rank 3-algebras above. On real spaces, such considerations are governed by the groups $\mathrm{SO}(n, m)$ with $n+m=6$. The central thread, however, focuses here on line and Complex geometry which finds its well-known counterparts in descriptions of electromagnetism and special relativity as well as - using transfer principles - in Dirac, gauge and quantum field theory. We discuss a simple picture where Complexe of second grade play the major and dominant rôle to unify (real) projective geometry, complex representation theory and line/Complex representations in order to proceed to dynamics.

PACS numbers: $02.20 .-\mathrm{a}, 02.40 .-\mathrm{k}, 03.70 .+\mathrm{k}, 04.20 .-\mathrm{q}$, 04.50.-h, 04.62.+v, 11.10.-z, 11.15.-q, 11.30.-j, 12.10.-g

\section{INTRODUCTION}

In [3] and [4] we have given arguments why and how to treat the Dirac algebra and various of its aspects in terms of groups on real, complex and quaternionic representation spaces. There, we have identified spin and isospin degrees of freedom within the compact group

\footnotetext{
* The author thanks the Alexander von Humboldt-Foundation (Bonn, Germany) for financial support.
} 
$\mathrm{SU}(4)\left(A_{3}\right)$ and it's representations, and we have related the non-compact group $\mathrm{SU} *(4)$, emerging from the complex embedding of quaternions, to the Dirac algebra via the identifications $\gamma^{0}=i \mathcal{Q}_{30}, \gamma^{j}=-i \mathcal{Q}_{2 j}$ and $\gamma_{5}=i \mathcal{Q}_{10}, Q_{\alpha \beta}$ denoting group elements, generators of the Lie algebra su*(4) or elements from the five-dimensional Riemannian space $\mathrm{SU} *(4) / \mathrm{USp}(4)$ or from the vector space $p, \operatorname{su} *(4)=h+p, h=\mathrm{usp}(4)$, according to the respective context(s). In [7], using the Lie algebras of [4] as an intermediary step towards projective and line geometry, we have extended the above approach, originally given in terms of (transformation) groups and their (Lie) algebras, by relating those algebras to a geometrical counterpart in terms of projective and especially line and Complex ${ }^{1}$ geometry.

Here, we want to summarize in section II briefly some of the major arguments developed so far and arrange them in a logic sequence in order to serve as a twofold basis for upcoming work and discussions: On one hand, we can reduce the group theoretical, algebraical and differential geometrical models which have been discussed over the years to their original content and concept which is based on nothing but Klein's Erlanger program. This means, a geometry is determined by the identification of states and the transformation groups, however, there exist various (geometrical) representations which can be (inter-)related using transfer principles. Practically, a single algebraic rep or equation can have different content and meaning depending e.g. on the interpretation of the coordinates involved. A lot of such representations and related transfer principles have been discussed in literature, we have mentioned some examples in [7] (see also references). Within this setting, we subsume complex representation theory and quantum field theory as subsets obtained by using appropriate complexifications of line geometry and projective (point and line) coordinates. On the other hand, it is known for more than a century (see e.g. [11] and references therein) how to derive euclidean, elliptic and hyperbolic geometry (and as such their transformation groups like a 'Lorentz' group, a 'Poincaré' group or a 'Galilei' group or homogeneous transformations on appropriate representation spaces as well) from projective geometry ${ }^{2}$.

That is why in section III we use a very simple picture which helps to illustrate and

${ }^{1}$ We use the terminology proposed in [7] which has been introduced by Plücker, and we denote by 'Complex' (with capital 'C') a line complex in order to avoid confusion with complex numbers.

${ }^{2}$ Here, we do not want to discuss more details or geometrical limites like contractions and expansions to relate homogeneous and affine transformations with respect to the 'Poincaré' group but we simply refer to [10], chapter 10 and references therein, as an overview and providing some examples and related algebra. 
understand the coincidence of various models and representations used so far. It is able to illustrate the concepts and derive physical aspects of dynamical systems for later use.

\section{ON THE ROAD TO PROJECTIVE AND LINE GEOMETRY}

Thinking of simple dynamical systems, the driving terms of almost all dynamical models are typically twofold: We choose a basic setup or description of the system in terms of one or more (then interacting or coupled) usually (irreducible) representations (later for short 'irreps') and assume a certain dynamical behaviour governed by certain group or algebra transformations, thus intrinsically assuming a 'well-behaved' nature of the dynamical system. The typical representation theory is thus based on Klein's Erlanger program, especially when using derivatives and analyticity to formulate the dynamics and system behaviour in terms of point manifolds and differential geometry. In this picture, the initial and the final state may both be characterized in terms of the chosen representation(s) (later 'reps') thus implicitly assuming a valid global coordinatization of the process ${ }^{3}$. Dynamics is described by applying a transformation (or a series of transformations completely described e.g. by Lie algebra generators or group actions) to the initial state, and applying the transformations is sufficient to 'reach' and characterize the final state in well-behaved rep spaces. Hence, in such scenarios the usual way to investigate dynamics by applying (Lie) group or algebra theory, invariant theory and appropriate algebraic concepts often is sufficient and helpful, especially when working with compact groups. However, non-compact groups and non-existing global coordinate systems complicate this algebraically founded approach considerably, and it helps to go back to the very foundations and use geometric pathways to reestablish physical reasoning and avoid some of the 'rep-only' or 'rep-induced' problems when having chosen not the best or most exact rep. The major (and almost the only) physical driving term is the terminology of an 'action' (or 'energy') - its formulation and conservation (or nonconservation) of energy - which allows to apply certain formalisms. In standard text books, this treatment is usually mapped to applying differential geometry in terms of Hamilton and/or Lagrange formalism and appropriate differential equations. Group schemes and applications considering energy as a conserved quantity usually apply representation theory

\footnotetext{
${ }^{3} \mathrm{Or}$ at least some knowledge on how to relate the coordinate systems used throughout the process/description.
} 
of compact groups to physical problems while identifying states with real or complex irreps thus introducing 'necessary' involutions (like complex conjugates, adjoints, hermiticity, ... ) and the related metrical properties by hand. Please note, that most of such properties from the viewpoint of projective geometry are derived features, i.e. they are already contained in projective geometry and they are derived typically by applying constraints on geometrical settings, objects, states and groups, e.g. by certain geometrical limites or identifications of elements and objects.

Now we do not argue to abandon this approach but it is well-known (although almost forgotten) that equivalent descriptions are possible, mostly due to the fact that Lie theory is a special (polar) and subsidiary concept of line and/or projective geometry in that we investigate tangents to a point manifold and their related dynamics, or 'line elements' as unions of 'points' and lines ${ }^{4}$. So instead of being concerned of point manifolds only (in terms of projective geometry: investigating the orders of the curves), we can as well look for the tangents more general as lines and their behaviour (in projective geometry e.g. by the classes of the curves or surfaces, by polar behaviour and duality or a possible projective generation of those objects) or even use and relate both pictures as has been suggested by Plücker e.g. in terms of his dimensional formulae [14]. A simple example has been referenced in [7] where the representation (or requirement) of a quadratic Complex automatically yields light cones when expressed in terms of point coordinates (of the manifold). In other words, representing physical objects by quadratic Complexe (i.e. Complexe of second grade) yields objects like (second-order) cones and 'light cones' in a manifold or point picture automatically, as has been structurally required by [9]. Please recall that typically light cones are introduced ad hoc by special relativity in conjunction with Weyl's gauge philosophy, and invariances and 'metrics' like the 'Lorentz metric' in special relativity require an 'extension' of the mathematical standard definition of a metric and a separate, non-standard treatment of negative metric values. This can be avoided using projective geometry and line (or better Complex) representations as well as Complex geometry. As such, Dirac's square root of the energy written in terms of momenta (for the free part) or in terms of line coordinates,

\footnotetext{
${ }^{4}$ In euclidean $\mathbb{R}^{3}$, we may represent such efforts by choosing 3 -dim point reps $\vec{x}=(x, y, z)$ whereas the line elements are represented by 5 -dim elements $(x, y, z, \mathrm{~d} x: \mathrm{d} y: \mathrm{d} z)$, i.e. it is the ratios of the elements $\mathrm{d} x, \mathrm{~d} y, \mathrm{~d} z$ which enter theory and describe the direction of the line intersecting the point $\vec{x}$; a concept which leads to Monge's and Pfaff's equations (see [7]).
} 
respectively, is a simple square of a line (or Complex) square, hence we find (linear 4-dim) transformation group reps acting on line reps [7. This yields exactly our statement above relating the Dirac matrices to linear reps of $\mathrm{SU} *(4)$ or $\mathrm{SO}(5,1)$, and we can use group and representation theory to perform the algebra and provide analytic reps. The 'potential' part may be formulated as well keeping in mind that we have to respect normals ${ }^{5}$, too, which can be included in line geometry using a simple euclidean/affine six-vector representation of a line which decomposes into a 3-dim polar vector and a 3-dim axial vector having different orders in terms of point coordinates (see e.g. [7, eq. (I.B (2)), and references therein). This, however, is achieved having chosen a special geometrical setup by fixing geometrical objects which at the same time restricts (real) projective transformations to orthogonal ones, i.e. we have introduced 'invariant' or 'fixed' (geometrical) objects with respect to the respective (coordinate) transformations. So we feel free to choose (from our viewpoint) better suited reps, and we understand point and manifold discussions subsequently as a subsidiary concept ${ }^{6}$ only. However, at any time it is possible to switch back to differential geometry and symmetric spaces [3].

From above, we follow the reasoning and interpretation of tangent 'objects' in order to map them on lines or Complexe. As an example, we use the global Riemannian space $\mathrm{SU} *(4) / \mathrm{USp}(4)$ (see [3], sec. 2, eq. (3)) with the rep

$$
X:=\exp V=\cosh \|x\| \mathbf{1}+\sinh \|x\| \frac{x_{a}}{\|x\|} \mathcal{P}_{a},
$$

where $\mathcal{P}_{a}, 1 \leq a \leq 5$, denote the five non-compact generators of the reductive Lie algebra decomposition of su*(4) when subtracting the ten (compact) usp(4) generators. Now with respect to the interpretation of the coset coordinates in eq. (1) we feel free to interpret the $\mathcal{P}_{a}$ as (generalized) velocity operators causing the symmetry transformations and to relate them to (infinitesimal) line representations ${ }^{7}$. The symmetry (or 'invariance') group has been

\footnotetext{
${ }^{5}$ Differential geometry uses gradients and (potential) functions to represent normal behaviour within the differential formalism by using the fact that a differential operator diminishes the power of a point/manifold coordinate by 1 . The same happens geometrically when introducing normal vectors by $n_{i} \sim \epsilon_{i j k} x_{i} y_{k}$, i.e. a (sub-)determinant. Please note however, that both reps above work only in affine or non-homogeneous euclidean coordinates! Hence the discussion of polar and axial vectors and 'parity' discussions at a first glance are related to this euclidean/affine picture only! A more sophisticated treatment can also investigate the Complex of normals of point curves or appropriate surfaces.

${ }^{6}$ Axiomatically, a point is fixed by incidence of two lines.

${ }^{7}$ Which, however, have to be identified only after having complexified some generators and compared to
} 
shown to be $\mathrm{SO}(5,1)$ which reflects in hyperbolic functions and the rank 1 , negatively curved 'space' ([3] and references).

From the viewpoint of dynamics in the underlying manifold, we may use the Lie algebra to develop the point motion by transforming 'point' and tangent (or the 'point velocity') with appropriate (Hamiltonian) symmetry constraints. On the other hand, we may take the viewpoint of Complexe (e.g., a (quadratic) tangential Complex or a tetrahedral Complex), abandon the construction of manifolds by points, continuity and analyticity, and instead switch to the associated notion of classes and envelopping structures of such a manifold where polarity and dimensional formulae enter. There exist, as we'll see in section III, also possibilities to construct or generate curves and surfaces in projective geometry. So discussing projective geometry of $\mathbb{R}^{3}$, (self-dual or conjugated) lines, Complexe and Complex geometry seem best suited as an underlying framework. There, it is well-known that a representation in terms of point/manifold coordinates has to be expressed by four (real) homogeneous coordinates whereas Complexe right from the beginning have different, even higher-dimensional reps in terms of (antisymmetric) line coordinates, and the condition of a quadratic Complex comprises automatically a light cone rep in point coordinates. Introducing a 'time' coordinate in the coordinate rep of the underlying point manifold (thus side-by-side the concept of a vectorial 'velocity' and the notion of dynamics in this local setup), we can identify c (interpreted as 'infinity' in velocity space) in the light cone definition, and by defining inhomogeneous/affine point coordinates we obtain ratios of velocities [7] (for overall/equal local time) which is consistent with Gilmore's presentation [10] of the typical coset constructions of representation spaces of transformation groups or symmetric spaces. The point coordinates themselves can be extracted only from this (euclidean) dynamical picture by introducing a transformation parameter $t$ common to all local coordinates $x_{i} \sim v_{i} t$ where $v_{i}$ are the transformation parameters or (generalized) velocities ${ }^{8}$. So

an $\mathrm{SO}(3,3)$ Plücker rep or the $\mathrm{SO}(6)$ Klein rep. In general, we can thus obtain the whole series $\mathrm{SO}(n, m)$, $n+m=6$, as can be seen from the signature of the squares. However, the respective interpretation of the coordinates has to be changed appropriately and carefully! Please note, that 'naive' complexification of the coordinates usually leads to $\mathrm{SU}(n, m), n+m=4$, but $\mathrm{SO}(5,1)$ is related to the quaternionic embedding $\mathrm{SU} *(4)$ and as such represents a special case.

${ }^{8}$ Or using, more generally, projective (4-dim) transformations relating different homogeneous coordinates $x_{\alpha}$ while avoiding an overall ' $t$ '. Anyhow, all approaches using linear 'time' have to reflect and represent 'velocity' equivalence classes from (special) relativity with respect to coordinates in projective geometry appropriately, and linear 'time' (parameter) dependence may be even replaced by more complicated 
from the viewpoint of an overall picture, we may use different $t$-values to enumerate different consecutive geometrical point sets of the dynamical system in the manifold. Hence we can label and relate the individual system states time $t_{n}$ by time $t_{n+1}$ from an overall (static) and purely geometrical setup while assuming appropriate transformations connecting the labeled states. The velocities (respectively their ratios) for the same time value reflect the rôle of building equivalence classes like the homogeneous coordinates of the point picture. This, on the other hand, is consistent with the group picture and the rep construction scheme using Lie algebras where one-parameter transformation groups transform and develop the physical/dynamical system [10].

\section{A SIMPLE PICTURE DEPARTING FROM POINT GEOMETRY}

For us, before going too much into detailed analytical discussions, a simple underlying picture departing from the usual point (or manifold) picture allows to attach and construct the various representations and models, if we start from nothing but two individual and distinguishable points $x_{1}$ and $y_{1}$ (see figure 1a).

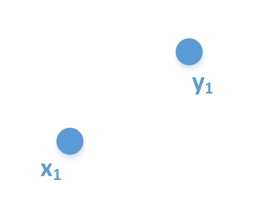

Figure 1a

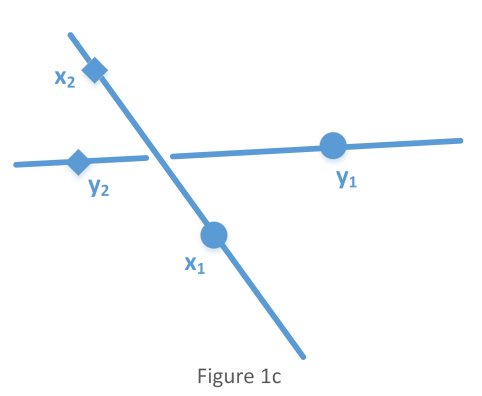

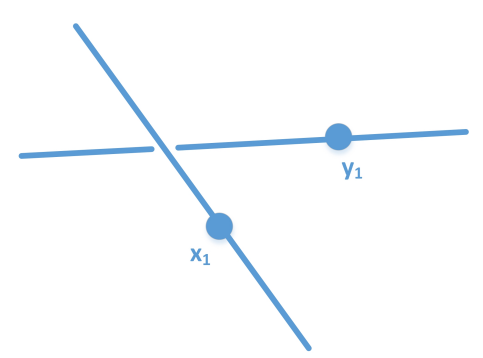

Figure $1 b$

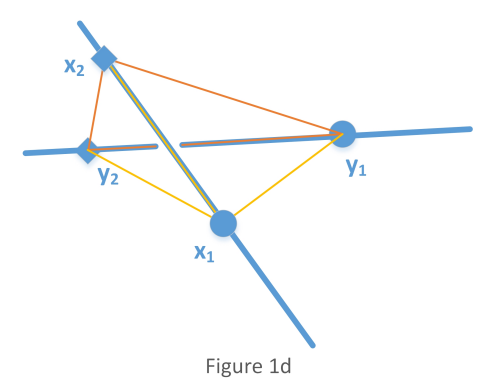

FIG. 1: Linear motion of points

Taking the points $x_{1}$ and $y_{1}$ for given (represented by circles) and transforming them

dependencies. 
individually by some (linear) action (figure 1b) to different points $x_{2}$ and $y_{2}$ (represented by diamonds), we may represent each connection of the two points in the point sets $x_{1} \longrightarrow x_{2}$ and $y_{1} \longrightarrow y_{2}$, generated by the (symmetry) transformation, by a line (figure 1c). Then already this very basic assumption comprises a lot of information and content in order to attach a wealth of formalism as we know from analysis and differential geometry.

Here however, and that's why we have drawn figure 1d, we gain a fortune in that the tetrahedron (which results when connecting the four points by lines) yields a foundation of connecting three (analytic) approaches we have used before only separately ${ }^{9}$.

\section{A. Projective Geometry of $\mathbb{R}^{3}$, Points and Coordinate Systems}

If we identify the four points $\left(x_{1}, x_{2}, y_{1}, y_{2}\right)$ of our picture individually, because having skew lines, no more than three points lie in each plane. Hence we are already very close to delivering a coordinate definition in projective 3 -space. The only additional information necessary to fix the coordinate system is an unit point $E$, and - having introduced such a point $E$ - we can proceed with well-known coordinate geometry, algebra and analysis [11]. The tetrahedron given in figure $1 \mathrm{~d}$ can be identified as the fundamental coordinate tetrahedron, and we may apply classical projective geometry with real transformations (or $4 \times 4$ real matrix reps, however, we have to take care about the transformation rep, e.g., its rank and further rep properties).

In order to lead over to the next subsection, we introduce an unit point $E$ in a manner that the coordinates of the four points $\left(x_{1}, x_{2}, y_{1}, y_{2}\right)$ in figure 1 are mapped to the coordinates $((1,0,0,0),(0,1,0,0),(0,0,1,0),(0,0,0,1))$. However, it is important to mention that alternatively instead of starting with the four points like before one can also identify the six sides of the tetrahedron and use them as line coordinates in a so-called six-vector rep of a line ${ }^{10}$.

\footnotetext{
${ }^{9}$ We are aware of richer mechanisms and content from projective geometry in that the two lines e.g. may be interpreted in terms of generators of a hyperboloid or general ruled surfaces or - associating e.g. focal properties - in terms of ray systems [12]. For now, we leave those details for upcoming discussions because the current focus is to supersede or unify the technical frameworks used so far.

10 Here once more, we could follow much deeper concepts from projective geometry e.g. by interpreting each of the two skew lines as an axis of a sheaf of planes thus intersecting the other line in a point series, i.e. we obtain this line being represented as point set with appropriate projective relations. Two conjugated lines (related by reciprocal polarity) lead to ray systems of $1^{\text {st }}$ order and class [12, [7] and to null systems. However, that's beyond scope here.
} 


\section{B. $\mathrm{SU}(4)$ and $\mathrm{SU} *(4)$}

Now in [1, appendix F.6 (see also [2]), we have constructed various representations of $\mathrm{SU}(4)$ which we may use immediately based on the above given points of the tetrahedron. Because $\mathrm{SU}(4)\left(A_{3}\right)$ is compact and of rank 3, we can transform the roots to a real 3-dim rep and draw pictures of the reps (see [1]). The fundamental rep $\underline{4}$ of SU(4) yields a tetrahedron, so we feel free to identify the root space diagram $\underline{4}$ with the fundamental tetrahedron of real projective 3-dim geometry and vice versa! Moreover, due to Young diagrammatics we may understand various representations as being build out of compositions of tetrahedra, a special case is the representation $\underline{\mathbf{2 0}}$ comprising four nucleon and sixteen delta degrees of freedom which we have discussed at various places before (see references). We have stated a surprising 'selfsimilarity' of the representations (see figure 21), and, sloppy speaking, $\underline{\mathbf{2 0}}$ is a 'cubic' of $\underline{4}$ due to the third-order symmetric tensor product ${ }^{11}$.
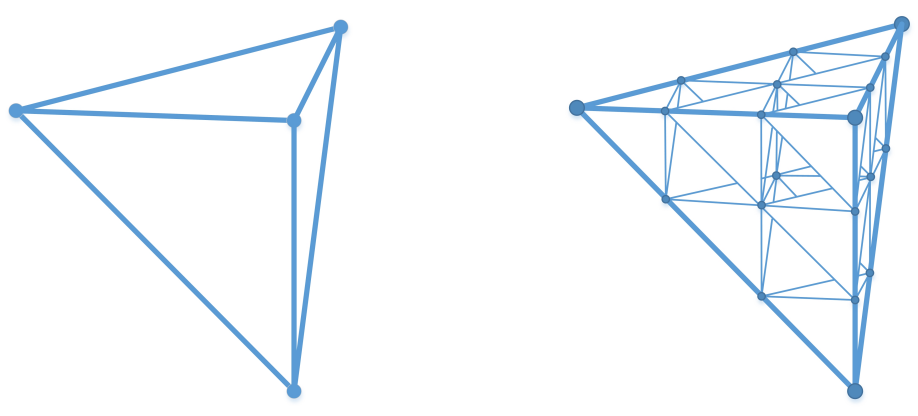

FIG. 2: SU(4) multiplets $\underline{4}$ and $\underline{\mathbf{2 0}}$

Acting with (general) complex transformations, of course, transforms the individual points of the tetrahedron $\underline{4}$ to general complex (4-dim) coordinates, however, we know that $\underline{4}$ and $\underline{20}$ both are irreps of su(4), so for very special choices of complex transformations (i.e. $\mathrm{su}(4)$ transformations ${ }^{12}$ ) we transform tetrahedra into themselves ${ }^{13}$. So obviously, here

${ }^{11}$ So with respect to $3^{\text {rd }}$-order curves, it is natural to expect (up to a common/overall normalization factor of the states) eigenvalues of \pm 1 and \pm 3 , see [1] and [7].

${ }^{12}$ Care has to be taken when acting with the group $\mathrm{SU}(4)$ because this involves anticommutators as well which complicates the situation considerably. Especially, also ternary products like Lie and Jordan triple systems (based on commutators and anticommutators) enter the scenario and yield additional information and constraints (see e.g. [3] and references), although the consequences are not independent from the underlying algebra or group.

13 A detailed discussion of the topics mentioned here has been given in [6] but due to some time problems this is not published yet. Therefore, a short summary is given in appendix A 
we can relate $\mathrm{SU}(4)$ Lie theory with (real) 3-dim projective geometry. To relate to noncompact $\mathrm{SU} *(4)$, we have to keep in mind that $\mathrm{SU} *(4)$ represents the complex embedding of quaternionic $\mathrm{SL}(2, \mathbb{H})$, and that we began by embedding two independent quaternions ('spin' and 'isospin' Lie algebras), i.e. two $A_{1}$ into $A_{3}$. Now with respect to such scenarios e.g. in elliptic geometry we have cited already some relevant literature (see references in [7]), here we want to emphasize only Study's work and Lie's line-sphere transformation as examples. The easiest interpretation at this time is to understand the so-called 'six-vector' of the line in terms of two 3-dim 'vectors' (its 'polar' and its 'axial' part) and require conservation of the 3-dim vector squares, even in the complex case ${ }^{14}$. SU*(4) on complex rep spaces or $\mathrm{SL}(2, \mathbb{H})$ as general real transformations of two real quaternions (and isomorphic to the Dirac algebra) thus can be used to represent certain geometrical transformations and behaviours, especially when mapped/transferred to line or projective geometry.

\section{Projective Geometry from Scratch}

It is even possible to look at the tetrahedron from a strictly synthetic viewpoint in terms of line geometry [17]. This can be easily seen by extending the basic planes of the tetrahedron in all spatial directions. So a general line ${ }^{15}$ in $\mathbb{R}^{3}$ will hit the planes in four different points. Now von Staudt showed [17] that the anharmonic ratio ${ }^{16}$ of the four intersection points of this line with the tetrahedral planes and that of the four planes each comprising this line and one of the four tetrahedral points is the same as long as the order of elements in the anharmonic ratio is the same ([17], Erstes Heft, $\S 2$, especially numbers 35 and 36). So the 'playground' is set for transformations respecting anharmonic ratios or investigations of the set of the $\infty^{1}$ anharmonic ratios, i.e. we are in the center of projective geometry. The more general theory of quadratic Complexe was developed in [14] or in Reye's 'reprise [15] (there especially part 2) of von Staudt's work.

Historically, and that's the basis to propose line geometry to describe dynamics as well,

14 This allows to represent and investigate typical properties of 3-dim 'vector' reps, of course, within its associated (euclidean) interpretation, 'vector' pictures like 'parity' and 'chirality' and appropriate counterparts when transferred to complex rep spaces e.g. in the context of $\mathrm{SL}(2, \mathbb{C})$. However, here we do not want to discuss such features or detailed reps of 'chiral symmetry'.

15 The line shouldn't lie in one of the planes and shouldn't hit one of the vertices of the tetrahedron.

${ }^{16}$ German: Wurf, Doppelverhältnis 
Complexe (and especially tetrahedral Complexe) appeared in various contexts. When considering the movement of rigid bodies around one of its points, the (infinitesimal) rotation axes build a (second-order) cone. Further examples are their Dreibeins of (orthogonal) axes of inertia which are attached to each point of the body and as well attached to each point of space when 'moving space' with the body, and also the related normals to confocal surfaces of second grade which were parametrized by

$$
\frac{x^{2}}{a^{2}+\lambda}+\frac{y^{2}}{b^{2}+\lambda}+\frac{z^{2}}{c^{2}+\lambda}=1
$$

$\lambda \in \mathbb{R}$ representing the 1 -dim parameter of the set of surfaces. And we may use quadratic Complexe as well to approach 'light cone' or sphere reps in point coordinates (see [7] for a reference to Plücker and work of Binet and Dupin). In plane coordinates $u, v, w$, eq. (2) reads as

$$
\left(a^{2}+\lambda\right) u^{2}+\left(b^{2}+\lambda\right) v^{2}+\left(c^{2}+\lambda\right) w^{2}=1 .
$$

Being linear in $\lambda$, we can attach a normal in the contact point of the surface with the plane, and we thus arrange/obtain a unique mapping of planes in space to normals. Similar investigations have been done for infinitesimal transformations, for the Complex of tangents related to (infinitesimal) motions and related second-grade cone(s). More general, we can map two (rigid) bodies pointwise onto each other, or extend this mapping to projectively mapping points in space [15]. If we use lines to connect the original points with their individual images, the lines constitute a tetrahedral Complex, so our picture in figure 1d above is obviously a subset of the general construction scheme. Moreover, considering normals of concentric second-order surfaces, which obey a constant anharmonic ratio with certain planes including the plane at infinity, leads to a tetrahedral Complex. This holds also for the normals of confocal surfaces of second order (see e.g. [17], [15] and references therein). We have mentioned already the well-known feature of e.g. Lorentz transformations to leave (affine euclidean) normal coordinates $x, y$ with respect to a $z$-axis of motion invariant [7]. This can be translated back to transformations of homogeneous or projective coordinates, normal planes (or null systems), or to the line coordinates themselves. So asking for invariance of certain ratios of homogeneous line coordinates or invariance of some line coordinates themselves leads to subsets of projective transformations and may be considered separately.

Having based our discussion so far on quadratic Complexe (i.e. Complexe of grade 2) and especially tangential and tetrahedral Complexe, on one hand, we feel very comfortable to 
apply the full framework of projective geometry, including dynamical treatment of points, second-order curves and surfaces, polar relationships, etc. in a more general and purely geometrical framework. On the other hand, this approach provides and satisfies the treatment of the requirements of [9] in a more general description than only using points and point manifolds. Of course, we are aware of transfer principles mapping objects like lines, Complexe or spheres to points in higher-dimensional spaces ${ }^{17}$, however, here we want to take up at first the 'classical' position of a real 3-dim projective geometry and mention the enormous wealth of this description (without switching to more complicated approaches which usually introduce additional ad hoc-assumptions or axioms on manifolds). So using [9] for the moment as a guideline to state the necessary (minimal) theoretical requirements of relativity, line and Complex geometry provide a unification basis for us with special emphasis on Complexe of first and second grade [14], [15].

Before closing this section, it should be mentioned that we may replace the straight lines in figure 1 by curves as well, see figure 3 . If the curves themselves respect further properties or obey further constraints, e.g. with respect to polar relations, null systems, conics with projective generation, Complex curves, intersections of higher-order surfaces, higher-order curves, etc., this enriches the given picture considerably but the treatment is completely possible within the approach presented here.

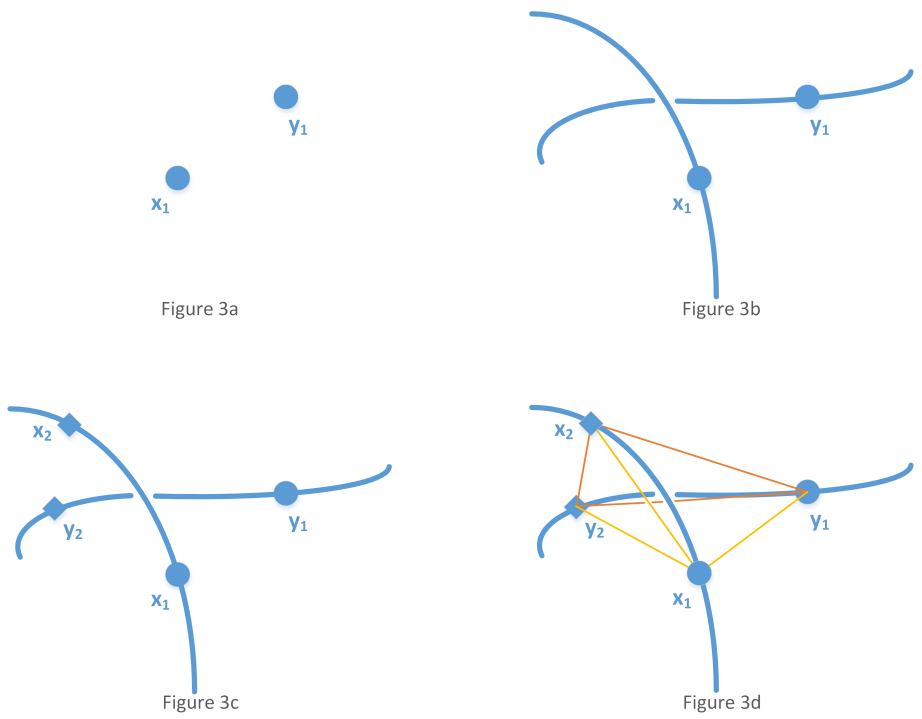

FIG. 3: Higher-order motion

17 E.g., the Plücker-Klein quadric, Laguerre geometry, cyclography, etc. 


\section{OUTLOOK}

After having presented various representations of projective geometry, our roadmap is fixed towards looking deeper into dynamics from the viewpoint of projective geometry with special emphasis on line and Complex geometry. We have mentioned few aspects already in the last section and in [7]. At the time of writing, we are convinced of having described and represented various aspects of projective geometry so far in terms of different group models and/or representations, so it is worth to look deeper into dynamics formulated in terms of line and Complex geometry. Last not least, we want to thank Bernd Schmeikal for deep and enlightening discussions at the Goslar conference 2015 and in Vienna in summer 2015, for his hospitality, interest and time there during my visit, and especially for his great work on logic based on fourfold base elements [16] which we want to see related to the two orientable lines or line elements in figure 1 (or figure 3). Moreover, it is a great pleasure to thank George Pogosyan and his team for the great conference athmosphere and organization in Yerevan and for the enormous hospitality while staying there.

\section{Appendix A: Short Remark on SU(4) Representations}

We want to summarize briefly some SU(4) rep facts as far as they are - to our opinion related to our reasoning with respect to projective geometry ${ }^{18}$.

The rank-3 group SU(4) (or $A_{3}$ ) has three fundamental representations $\square$ or $[1,0,0]$,<smiles>[CH]1CCC1</smiles>

or $[1,1,0]$, and

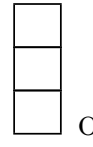

or $[1,1,1]$, of dimensions 4,6 and 4 , respectively, (see e.g. [1], appendix F.6, or [13], p. 99/100) and we may perform standard transformation operations with the reps above or construct interactions or construct invariants etc. Now from Young diagrammatics, we know that the reps are antisymmetric with respect to vertical rows (due to the permutation group construction scheme). Moreover, we know that $[1,1,0]$ is selfconjugate and of $\operatorname{dim} 6$ whereas $[1,0,0]$ and $[1,1,1]$ (each of dim 4) are mapped to each other by conjugation, and we thus work with an involution.

Now identifying $\underline{\mathbf{4}} \sim[1,0,0]$ with a point rep, the antisymmetric 'products' of point reps in case of $\sim[1,1,0]$ are equivalent to the very definition of a line rep (see [7] for the analytic

${ }^{18}$ Conference talk at QTS 7, Prague, 2011, publication upcoming soon 
expression) whereas $\sim[1,1,1]$ maps to a third-order (sub-)determinant which complies to the 'standard definition' of plane coordinates $u_{\alpha}, 0 \leq \alpha \leq 3$, in terms of (projective) 4-dim point coordinates of $\mathbb{R}^{3}$, i.e. we find natural associations to $\underline{\mathbf{6}}$ and the second $\underline{\mathbf{4}}$. Whereas the higher-order products can by calculated/represented by Young diagrammatics, here as a second approach towards the background rep theory, we want to mention a special, but established aspect of projective geometry (see [8], I §3, Nr. 8), valid for general polyhedrons with respect to duality in 3-dim space, here however applied with respect to the tetrahedron. There, denoting by $e$ vertices, $f$ areas and $k$ edges, the dual (or 'reciprocal') polyhedron consist of $e$ areas, $f$ vertices and $k$ edges. Now, in the case of the tetrahedron, we thus find/recover the mappings $4 \longleftrightarrow 4$ and $6 \circlearrowleft$, which reflect in the three fundamental reps $\underline{4}$, $\underline{\mathbf{4}}$ and $\underline{\mathbf{6}}$, and emphasize their identification with point-, area- and line-reps (edges).

So we feel authorized to use projective (3-dim) geometry from scratch, and subsume the group theoretical approaches and reps to cover certain analytical facets thereof. As with respect to the usual discussion of complex numbers we point to the LONG discussion in (geometric) literature and with respect to geometric interpretations. And no, we do not want to discuss and understand complex numbers only from the (contaminated) viewpoint of complex analyticity and differential geometry.

Last not least, we want to point to the use of the background discussed above in the case of self-polar tetrahedra in coordinate systems and especially in line and Complex geometry (see e.g. [11]) as well as the well-known context of [12] to focal surfaces which we can relate immediately to vertices in QFT. The very definition of a focal surface ([12], p. 5) yields the definition of a standard vertex (1 line or momentum $\longleftrightarrow 2$ lines or momenta), so for $n=2$ and $k=2$ (i.e. ray systems of order 2 and class 2 ) we may apply this framework in that we identify a QFT vertex as being a point of the focal surface and proceed with line/Complex geometry instead of (sometimes) mysterious 'quantum' argumentation in terms of point manifolds ${ }^{19}$.

19 Reading [12], it is noteworthy to point to theorems $33(\S 7)$ and $38(\S 8)$ for later use as well as to emphasize the common ground with Jacobi's and Hamilton's classical approaches to dynamics. 


\section{References}

[1] Dahm R 1996. Spin-Flavour-Symmetrien und das $\pi N \Delta$-System. (Aachen: Shaker Verlag)

[2] Dahm R 1995. Relativistic SU(4) and Quaternions. Adv. in Applied Clifford Algebra 7(S) 337

[3] Dahm R 2010. A Symmetry Reduction Scheme of the Dirac Algebra without Dimensional Defects. Yad. Fis. 73 297; Phys. Atom. Nuclei 73276

[4] Dahm R 2012. On A Microscopic Representation of Space-Time. Yad. Fis. 75 1244; Phys. Atom. Nuclei 75 1173; http://arxiv.org/abs/1102.0027

[5] Dahm R 2011. On A Microscopic Representation of Space-Time II. ICCA 9, Weimar, to be published

[6] Dahm R 2011 Projective Geometry, Conformal and Lie Symmetries and Their Breakdown to

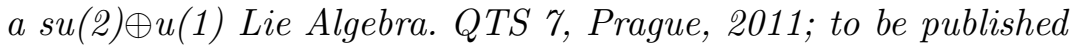

[7] Dahm R 2015. On A Microscopic Representation of Space-Time III. ICGT 30, Ghent and ICCA 10, Tartu, http://arxiv.org/abs/1508.06872, submitted to AACA

[8] Doehlemann K 1905. Projektive Geometrie in synthetischer Behandlung. (Sammlung Göschen) (3rd edition, Leipzig: G. J. Göschen'sche Verlagshandlung) (1898)

[9] Ehlers J, Pirani F and Schild A 1972. The Geometry of Free Fall and Light Propagation. In: Studies in Relativity. ed L O'Raifeartaigh (Oxford: Clarendon Press) pp 63-84

[10] Gilmore R 1974. Lie Groups, Lie Algebras and Some of Their Applications. (New York: John Wiley \& Sons)

[11] Klein F 1928. Vorlesungen über nicht-euklidische Geometrie. (Die Grundlehren der mathematischen Wissenschaft XXVI) (Berlin: Springer)

[12] Kummer E E 1866. Über die algebraischen Strahlensysteme, in's Besondere über die der ersten und zweiten Ordnung. Abh. Akad. Wiss. Berlin, Math. Abhandlungen, 1

[13] Lichtenberg D B 1970. Unitary Symmetries and Elementary Particles. (Academic Press)

[14] Plücker J 1868/1869. Neue Geometrie des Raumes. ed A Clebsch and F Klein (Leipzig: B. G. Teubner)

[15] Reye Th 1899. Die Geometrie der Lage I-III. (4th edition, Leipzig: Baumgärtners Buchhandlung) (1866/1868) 
[16] Schmeikal B 2015. Four Forms Make A Universe, Adv. in Applied Clifford Algebra 25 (1), 1 (2015), DOI10.1007/s00006-015-0551-z, and private communication

[17] von Staudt G K C 1856. Beiträge zur Geometrie der Lage. (Nürnberg: Bauer und Raspe) 